\title{
Completely replicable functions and symmetries
}

\author{
Bernhard Heim* and Atsushi Murase ${ }^{\dagger}$
}

August 18, 2019

\begin{abstract}
Completely replicable functions play an important role in number theory and finite group theory, in particular the Monstrous Moonshine. In this paper, we give a characterization of completely replicable functions by certain symmetries.
\end{abstract}

Keywords: completely replicable functions, Faber polynomials, symmetries.

Mathematics Subject Classification 2010: 11F03, 11F12, 11F22,

\section{Introduction}

Conway and Norton ([CN]) introduced the replication formula for the modular invariant $J(q)=q^{-1}+196884 q+21493760 q^{2}+\cdots$ together with the conjectural head characters of the monster in the formulation of Monstrous Moonshine conjecture. Later Norton ([N]) introduced the notion of replicability and complete replicability for a formal Laurent series $f(q)=q^{-1}+\sum_{m=1}^{\infty} H_{m} q^{m}$. Completely replicable functions play an important role in the proof of the Monstrous Moonshine conjecture by Borcherds ([Bo]).

Norton ([N]) stated that a (partly) completely replicable function satisfies certain symmetries, which we generalize and call multiplicative symmetries (see Remark 2.5). In this paper, we prove that these symmetries already characterize completely replicable functions.

*Department of Mathematics and Science, Faculty of Science, German University of Technology in Oman, Muscat, Sultanate of Oman; e-mail: bernhard.heim@gutech.edu.om

${ }^{\dagger}$ Faculty of Science, Kyoto Sangyo University, Motoyama, Kamigamo, Kita-ku, 603-8555 Kyoto, Japan; e-mail: murase@cc.kyoto-su.ac.jp (corresponding author) 
We recall the definition of the bivarial transform $\left\{H_{m, n}\right\}_{m, n \geq 1}$ of a formal Laurent series $f=q^{-1}+\sum_{m=1} H_{m} q^{m}\left(H_{m} \in \mathbb{C}\right)$ given by

$$
-\log \left(\frac{f(q)-f(r)}{q^{-1}-r^{-1}}\right)=\sum_{m, n \geq 1} H_{m, n} q^{m} r^{n} .
$$

We say that $f$ is replicable if the equality $H_{m, n}=H_{m^{\prime}, n^{\prime}}$ holds for $(m, n),\left(m^{\prime}, n^{\prime}\right) \in$ $\left(\mathbb{Z}_{>0}\right)^{2}$ satisfying $m n=m^{\prime} n^{\prime}$ and $\operatorname{gcd}(m, n)=\operatorname{gcd}\left(m^{\prime}, n^{\prime}\right)$. For $s \geq 1$, we set $H_{m}^{(s)}:=$ $s \sum_{j \mid s} \mu(j) H_{j^{-1} s, j s m}$, where $\mu$ stands for the Möbius function. We define the $s$-th replicate $f^{(s)}$ of $f$ by $f^{(s)}(q)=q^{-1}+\sum_{m \geq 1} H_{m}^{(s)} q^{m}$. We say that $f$ is completely replicable if $f^{(s)}$ is replicable for any $s \geq 1$ and the equality $\left(f^{(s)}\right)^{(t)}=f^{(s t)}$ holds for any $s, t \geq 1$.

We say that $f$ satisfies multiplicative symmetries if

$$
\prod_{\substack{a d=N \\ 0 \leq b<d}}\left(f^{(a s)}(q)-f^{(a s)}\left(\zeta_{d}^{b} r^{a / d}\right)\right)
$$

is symmetric or anti-symmetric in $q$ and $r$ for any $s, N \geq 1$. Here $\zeta_{d}:=\exp (2 \pi i / d)$.

The main result of the paper says that $f$ is completely replicable if and only if $f$ satisfies multiplicative symmetries.

The paper is organized as follows. In Section 2, we recall the definitions of replicability, completely replicability and multiplicative symmetries. In Section 3, we state and prove the main result of the paper. In Section 4, we make some comments.

\section{Complete replicability and multiplicative symmetries}

Throughout this section, we let

$$
f(q)=q^{-1}+\sum_{m=1}^{\infty} H_{m} q^{m}
$$

be a formal Laurent series in $q$ with coefficients in $\mathbb{C}$.

\subsection{Bivarial transforms}

Set

$$
\begin{aligned}
F(f ; q, r) & :=-\log \frac{f(q)-f(r)}{q^{-1}-r^{-1}} \\
& =-\log \left(1-q r \sum_{m=1}^{\infty} H_{m} \frac{q^{m}-r^{m}}{q-r}\right),
\end{aligned}
$$


where $q$ and $r$ are indeterminates. We expand $F(f ; q, r)$ into a formal power series in $q$ and $r$ :

$$
F(f ; q, r)=\sum_{m, n=1}^{\infty} H_{m, n} q^{m} r^{n}
$$

and call the sequence $\left\{H_{m, n}\right\}_{m, n \geq 1}$ the bivarial transform of $f$ or $\left\{H_{m}\right\}_{m \geq 1}([\mathrm{~N}])$.

\subsection{Replicates}

For $s \in \mathbb{Z}_{>0}$, we define the s-th replicate $f^{(s)}$ of $f$ by

$$
f^{(s)}(q):=q^{-1}+\sum_{m=1}^{\infty} H_{m}^{(s)} q^{m}
$$

where

$$
H_{m}^{(s)}:=s \sum_{j \mid s} \mu(j) H_{j^{-1} s, j s m} .
$$

Note that $f^{(1)}=f$ since $H_{m}^{(1)}=H_{1, m}=H_{m}$.

\subsection{Faber polynomials}

For $m \in \mathbb{Z}_{>0}$, there exists a unique polynomial $P_{f, m}(X)$ in $X$ such that $P_{f, m}(f(q)) \in$ $q^{-m}+q \mathbb{C}[[q]]([\mathrm{Fa}])$. For example, we have

$$
P_{f, 1}(X)=X, P_{f, 2}(X)=X^{2}-2 H_{1}, P_{f, 3}(X)=X^{3}-3 H_{1} X-3 H_{2} .
$$

We call $P_{f, m}(X)$ the $m$-th Faber polynomial attached to $f$. The following result is easily checked (see [Schi] for example).

Lemma 2.1. We have

$$
\log (q(f(q)-X))=-\sum_{m=1}^{\infty} \frac{1}{m} P_{f, m}(X) q^{m}
$$

and

$$
P_{f, m}(f(q))=q^{-m}+m \sum_{n=1}^{\infty} H_{m, n} q^{n}
$$

\subsection{Replicable functions}

We say that $f(q)$ is replicable if the equality $H_{m, n}=H_{m^{\prime}, n^{\prime}}$ holds for any $(m, n),\left(m^{\prime}, n^{\prime}\right) \in$ $\left(\mathbb{Z}_{>0}\right)^{2}$ satisfying $m n=m^{\prime} n^{\prime}$ and $\operatorname{gcd}(m, n)=\operatorname{gcd}\left(m^{\prime}, n^{\prime}\right)([\mathrm{N}]$; see also [Cu, Ma]). It is known that

$$
f(q) \text { is replicable } \Longleftrightarrow P_{f, N}(f(q))=\sum_{\substack{a d=N \\ 0 \leq b<d}} f^{(a)}\left(\zeta_{d}^{b} q^{a / d}\right) \quad \text { for any } N \in \mathbb{Z}_{\geq 0}
$$


(for example see [ACMS]).

Remark 2.2. Let $f(q)=q^{-1}+\sum_{m=1}^{M} H_{m} q^{m}$ be a finite Laurent series. Cummins and Norton ([CN]) showed that $f(q)$ is replicable if and only if $f(q)=q^{-1}+H_{1} q$ (namely $H_{m}=0$ for $\left.m \geq 2\right)$. In this case, we have $f^{(s)}(q)=q^{-1}+H_{1}^{s} q$.

\subsection{Completely replicable functions}

We say that $f(q)$ is completely replicable if the s-th replicate $f^{(s)}$ of $f$ is replicable for any $s \geq 1$ and the equality $\left(f^{(s)}\right)^{(t)}=f^{(s t)}$ holds for any $s, t \geq 1$ (see [N] and [K2]). The monstrous moonshine functions are completely replicable ([Bo]). For the definition of completely replicable functions, we also refer to [Ca], Section 5, Note.

Remark 2.3. In view of Remark 2.2, $f(q)=q^{-1}+H_{1} q$ is completely replicable.

\subsection{Multiplicative symmetries}

We say that $f$ satisfies multiplicative symmetries if the equality

$$
\prod_{\substack{a d=N \\ 0 \leq b<d}}\left(f^{(a s)}(q)-f^{(a s)}\left(\zeta_{d}^{b} r^{a / d}\right)\right)=\epsilon_{N} \prod_{\substack{a d=N \\ 0 \leq b<d}}\left(f^{(a s)}\left(\zeta_{d}^{b} q^{a / d}\right)-f^{(a s)}(r)\right)
$$

holds for any $s, N \geq 1$ with $\epsilon_{N} \in \mathbb{C}^{\times}$. It is easily seen that $\epsilon_{N}=\prod_{d \mid N} \zeta_{d}^{d(d-1) / 2}=$ $(-1)^{\sum_{d \mid N}(d-1)}$ if $(2.9)$ holds.

Remark 2.4. If (2.9) holds for any $s \geq 1$ and $N=p, p$ any prime, then $f$ satisfies multiplicative symmetries.

Remark 2.5. The multiplicative symmetries was first introduced by Norton in [N]. He stated (without proof) that, for a prime $p$,

$$
\left(f^{(p)}\left(q^{p}\right)-f^{(p)}(r)\right) \prod_{b=0}^{p-1}\left(f\left(\zeta_{p}^{b} q^{1 / p}\right)-f(r)\right)
$$

is symmetric in $q$ and $r$ if $f$ and $f^{(p)}$ are replicable.

\section{The main result}

\subsection{The main theorem}

We now state the main result of the paper. 
Theorem 3.1. Let $f(q)=q^{-1}+\sum_{m=1}^{\infty} H_{m} q^{m}\left(H_{m} \in \mathbb{C}\right)$. Then $f$ is completely replicable if and only if $f$ satisfies multiplicative symmetries (2.9) with $\epsilon_{N}=(-1)^{\sum_{d \mid N}(d-1)}$.

From now on, $f^{(s)}(q)=q^{-1}+\sum_{m=1}^{\infty} H_{m}^{(s)} q^{m}$ denotes the s-th replicate of $f(q)$, and $\left\{H_{m, n}^{(s)}\right\}_{m, n \geq 1}$ the bivarial transform of $f^{(s)}$.

\subsection{Preparations}

Let $X_{1}, X_{2}, \ldots$ be indeterminates. We define a family $\left\{\mathcal{G}_{m}\left(X_{1}, \ldots, X_{m}\right)\right\}_{m=1}^{\infty}$ of polynomials in $X_{1}, X_{2}, \ldots$ by

$$
\log \left(1+\sum_{m=1}^{\infty} X_{m} q^{m}\right)=\sum_{m=1}^{\infty} \mathcal{G}_{m}\left(X_{1}, \ldots, X_{m}\right) q^{m}
$$

The following result is a direct consequence of (2.6) and (3.1).

Lemma 3.2. We have

$$
P_{f, m}(X)=-m \mathcal{G}_{m}\left(-X, H_{1}, \ldots, H_{m-1}\right)
$$

For $d \in \mathbb{Z}_{>0}$, we set

$$
R_{d}\left(t ; X_{1}, X_{2}, \ldots\right)=\prod_{b=0}^{d-1}\left(1+\sum_{m=1}^{\infty} \zeta_{d}^{b m} X_{m} t^{m}\right) .
$$

Later we need the following elementary fact.

Lemma 3.3. We have

$$
R_{d}\left(t ; X_{1}, X_{2}, \ldots\right) \in 1+d \mathcal{G}_{d}\left(X_{1}, \ldots, X_{d}\right) t^{d}+t^{2 d} \mathbb{C}[[t]]\left[X_{1}, X_{2}, \ldots\right]
$$

Proof. In view of (3.1), we have

$$
\begin{aligned}
R_{d}\left(t ; X_{1}, X_{2}, \ldots\right) & =\exp \left(\sum_{b=0}^{d-1} \sum_{m=1}^{\infty} \mathcal{G}_{m}\left(X_{1}, \ldots, X_{m}\right) \zeta_{d}^{b m} t^{m}\right) \\
& =\exp \left(d \sum_{m=1}^{\infty} \mathcal{G}_{d m}\left(X_{1}, \ldots, X_{d m}\right) t^{d m}\right)
\end{aligned}
$$

which implies the assertion of the lemma.

Proposition 3.4. The following three conditions are equivalent:

(1) $f$ is completely replicable. 
(2) The equality

$$
\sum_{\substack{a d=N \\ 0 \leq b<d}} f^{(a s)}\left(\zeta_{d}^{b} q^{a / d}\right)=-N \mathcal{G}_{N}\left(-f^{(s)}(q), H_{1}^{(s)}, \ldots, H_{N-1}^{(s)}\right)
$$

holds for any s, $N \geq 1$.

(3) We have $H_{m, n}^{(s)}=\sum_{t \mid \operatorname{gcd}(m, n)} t^{-1} H_{t^{-2} m n}^{(s t)}$ for any $s \geq 1$ and any $(m, n) \in\left(\mathbb{Z}_{>0}\right)^{2}$.

Proof. This fact is elementary. We present its proof for completeness.

In view of (2.8) and Lemma 3.2, (1) implies (2).

Next suppose that (2) holds. The left-hand side of (3.3) equals

$$
q^{-N}+N \sum_{m=1}^{\infty} \sum_{a \mid \operatorname{gcd}(m, N)} a^{-1} H_{a^{-2} m N}^{(a s)} q^{m}
$$

On the other hand, the right-hand side of (3.3) equals

$$
P_{N, f^{(s)}}\left(f^{(s)}(q)\right)=q^{-N}+N \sum_{m=1}^{\infty} H_{N, m}^{(s)} q^{m}
$$

by Lemma 3.2 and (2.7). It follows that

$$
H_{N, m}^{(s)}=\sum_{a \mid \operatorname{gcd}(m, N)} a^{-1} H_{a^{-2} m N}^{(a s)} \quad(N, m \geq 1),
$$

which implies (3).

Finally suppose that (3) holds. We then have

$$
m n=m^{\prime} n^{\prime} \text { and } \operatorname{gcd}(m, n)=\operatorname{gcd}\left(m^{\prime}, n^{\prime}\right) \Longrightarrow H_{m, n}^{(s)}=H_{m^{\prime}, n^{\prime \prime}}^{(s)}
$$

which implies that $f^{(s)}(q)$ is replicable for any $s \geq 1$. Furthermore we have

$$
\begin{aligned}
t \sum_{j \mid t} \mu(j) H_{j^{-1} t, j t m}^{(s)} & =t \sum_{j \mid t} \mu(j) \sum_{k \mid j^{-1} t} k^{-1} H_{k^{-2} t^{2} m}^{(k s)} \\
& =t \sum_{k \mid t} k^{-1} H_{k^{-2} t^{2} m}^{(k s)} \sum_{j \mid k^{-1} t} \mu(j) \\
& =H_{m}^{(s t)},
\end{aligned}
$$

which implies $\left(f^{(s)}\right)^{(t)}=f^{(s t)}$. We thus have proved that $f(q)$ is completely replicable. 


\subsection{The proof of the "if"-part of Theorem 3.1}

Suppose that $f$ satisfies multiplicative symmetries. Denote by $\sigma(N)$ the sum of the positive divisors of N. By Lemma 3.3, we have

$$
\begin{aligned}
& \epsilon_{N} q^{\sigma(N)} \prod_{\substack{a d=N \\
0 \leq b<d}}\left(f^{(a s)}\left(\zeta_{d}^{b} q^{a / d}\right)-f^{(a s)}(r)\right) \\
& =\prod_{\substack{a d=N \\
0 \leq b<d}}\left(1-\zeta_{d}^{b} q^{a / d} f^{(a s)}(r)+\sum_{m=2}^{\infty} H_{m-1}^{(a s)} \zeta_{d}^{b m} q^{a m / d}\right) \\
& =\prod_{a d=N} R_{d}\left(q^{a / d} ;-f^{(a s)}(r), H_{1}^{(a s)}, H_{2}^{(a s)}, \ldots,\right) \\
& =\prod_{a d=N}\left\{1+d \mathcal{G}_{d}\left(-f^{(a s)}(r), H_{1}^{(a s)}, \ldots, H_{d-1}^{(a s)}\right) q^{a}+O\left(q^{2 a}\right)\right\} \\
& =1+N \mathcal{G}_{N}\left(-f^{(s)}(r), H_{1}^{(s)}, \ldots, H_{N-1}^{(s)}\right) q+O\left(q^{2}\right) .
\end{aligned}
$$

On the other hand, we have

$$
\begin{aligned}
& q^{\sigma(N)} \prod_{\substack{a d=N \\
0 \leq b<d}}\left(f^{(a s)}(q)-f^{(a s)}\left(\zeta_{d}^{b} r^{a / d}\right)\right) \\
& =\prod_{\substack{a d=N \\
0 \leq b<d}}\left(1-f^{(a s)}\left(\zeta_{d}^{b} r^{a / d}\right) q+O\left(q^{2}\right)\right) \\
& =1-\sum_{\substack{a d=N \\
0 \leq b<d}} f^{(a s)}\left(\zeta_{d}^{b} r^{a / d}\right) q+O\left(q^{2}\right) .
\end{aligned}
$$

Comparing the coefficients of $q$, we obtain

$$
\sum_{\substack{a d=N \\ 0 \leq b<d}} f^{(a s)}\left(\zeta_{d}^{b} r^{a / d}\right)=-N \mathcal{G}_{N}\left(-f^{(s)}(r), H_{1}^{(s)}, \ldots, H_{N-1}^{(s)}\right),
$$

which implies that $f$ is completely replicable by Proposition 3.4.

Remark 3.5. A similar argument shows that, if $f$ satisfies (2.9) for $s=1$ and any $N \geq 1$, then $f$ is replicable. The converse is not necessarily true (see Section 4 ).

\subsection{The proof of the "only if"-part of Theorem 3.1}

Suppose that $f$ is completely replicable. By Proposition 3.4, we have

$$
H_{m, n}^{(s)}=\sum_{t \mid \operatorname{gcd}(m, n)} t^{-1} H_{t^{-2} m n}^{(s t)} \quad\left(s \geq 1,(m, n) \in\left(\mathbb{Z}_{>0}\right)^{2}\right) .
$$


For $s, N \geq 1$, we set

$$
A_{N}^{(s)}(q, r)=\log \left(\prod_{\substack{a d=N \\ 0 \leq b<d}} \frac{f^{(a s)}\left(\zeta_{d}^{b} q^{a / d}\right)-f^{(a s)}(r)}{\left(\zeta_{d}^{b} q^{a / d}\right)^{-1}-r^{-1}}\right)
$$

Since

$$
\prod_{\substack{a d=N \\ 0 \leq b<d}}\left(\left(\zeta_{d}^{b} q^{a / d}\right)^{-1}-r^{-1}\right)=\epsilon_{N} \prod_{a d=N}\left(q^{-a}-r^{-d}\right)
$$

it is sufficient to show the symmetry of $A_{N}^{(s)}(q, r)$ in $q$ and $r$ for any $s, N \geq 1$. For $s, N, m, n \geq 1$, set

$$
\mathcal{H}_{N}^{(s)}(m, n):=\sum_{k \mid \operatorname{gcd}(m, N)} k^{-1} H_{m N / k^{2}, n}^{(k s)}
$$

We have

$$
\begin{aligned}
A_{N}^{(s)}(q, r) & =-\sum_{\substack{a d=N \\
0 \leq b<d}} F\left(f^{(a s)} ; \zeta_{d}^{b} q^{a / d}, r\right) \\
& =-\sum_{\substack{a d=N \\
0 \leq b<d}} \sum_{m, n=1}^{\infty} H_{m, n}^{(a s)}\left(\zeta_{d}^{b} q^{a / d}\right)^{m} r^{n} \\
& =-\sum_{a d=N} d \sum_{m, n=1}^{\infty} H_{d m, n}^{(a s)} q^{a m} r^{n} \\
& =-N \sum_{m, n=1}^{\infty}\left(\sum_{a \mid \operatorname{gcd}(m, N)} a^{-1} H_{m N / a^{2}, n}^{(a s)}\right) q^{m} r^{n} \\
& =-N \sum_{m, n=1}^{\infty} \mathcal{H}_{N}^{(s)}(m, n) q^{m} r^{n} .
\end{aligned}
$$

It now remains to show the symmetry of $\mathcal{H}_{N}^{(s)}(m, n)$ in $m$ and $n$, which follows from the next two lemmas.

Lemma 3.6. We have

$$
\mathcal{H}_{N}^{(s)}(m, n)=\sum_{(k, l) \in X_{N}(m, n)}(k l)^{-1} H_{(k l)^{-2} m n N}^{(k l s)}
$$

Here

$$
X_{N}(m, n):=\left\{(k, l) \in\left(\mathbb{Z}_{>0}\right)^{2}|k| \operatorname{gcd}(m, N), l \mid \operatorname{gcd}\left(\frac{m N}{k^{2}}, n\right)\right\} .
$$

Proof. This follows from (3.4). 
Lemma 3.7. For $(k, l) \in X_{N}(m, n)$, we let

$$
\varphi(k, l):=\left(\operatorname{gcd}(l, N / k), \frac{k l}{\operatorname{gcd}(l, N / k)}\right) .
$$

Then $\varphi$ defines a bijection from $X_{N}(m, n)$ to $X_{N}(n, m)$.

Proof. Let $(k, l) \in X_{N}(m, n)$ and set $(\kappa, \lambda):=\varphi(k, l)$. We first show that $(\kappa, \lambda) \in$ $X_{N}(n, m)$. Since $\kappa \mid l$ and $l \mid n$, we have $\kappa \mid n$ and hence $\kappa \mid \operatorname{gcd}(n, N)$. It follows from $k l \mid m l$, $k l \mid(m N / k)$ and $\lambda=k l / \kappa$ that $\kappa \lambda$ divides $\operatorname{gcd}(m l, m N / k)=m \kappa$ and hence $\lambda \mid m$. Since $\frac{n N}{\kappa^{2} \lambda}=\frac{k^{-1} N}{\kappa} \frac{n}{l} \in \mathbb{Z}$, we have $\lambda \mid \operatorname{gcd}\left(n N / \kappa^{2}, m\right)$. Thus $(\kappa, \lambda) \in X_{N}(n, m)$ and hence $\varphi$ defines a mapping from $X_{N}(m, n)$ to $X_{N}(n, m)$. It is straightforward to see that

$$
\psi(\kappa, \lambda):=\left(\operatorname{gcd}(\lambda, N / \kappa), \frac{\kappa \lambda}{\operatorname{gcd}(\lambda, N / \kappa)}\right) \quad((\kappa, \lambda) \in X(n, m))
$$

is a mapping from $X_{N}(n, m)$ to $X_{N}(m, n)$ and that $\psi \circ \varphi$ (respectively $\varphi \circ \psi$ ) is the identity map on $X_{N}(m, n)$ (respectively $X_{N}(n, m)$ ). This completes the proof of the lemma.

\section{Comments}

1. We give an example of a replicable function which does not satisfy multiplicative symmetries and hence is not completely replicable (this example is given in [N]).

Let $J(q)=q^{-1}+\sum_{m=1}^{\infty} H_{m} q^{m}\left(H_{1}=196884, H_{2}=21493760, \ldots\right)$ be the modular invariant and let $g(q)=-J(-q)=q^{-1}+\sum_{m=1}^{\infty} G_{m} q^{m}\left(G_{m}=(-1)^{m+1} H_{m}\right)$. Denote by $\left\{H_{m, n}\right\}$ (respectively $\left\{G_{m, n}\right\}$ ) the bivarial transform of $J$ (respectively $g$ ). Since $G_{m, n}=(-1)^{m+n} H_{m, n}$ and $J$ is replicable, $g$ is also replicable. We have $g^{(2)}(q)=$ $q^{-1}+\sum_{m=1}^{\infty} G_{m}^{(2)} q^{m}$ with $G_{m}^{(2)}=2 H_{4 m}+2 H_{2 m+1}+\sum_{j=1}^{2 m-1} H_{j} H_{2 m-j}$. A numerical computation shows that (2.9) does not hold for $f=g, s=1$ and $N=2$. It follows that $g$ is not completely replicable. This also shows that replicability for $f$ does not necessarily imply multiplicative symmetries for $s=1$ :

$$
\prod_{\substack{a d=N \\ 0 \leq b<d}}\left(f^{(a)}(q)-f^{(a)}\left(\zeta_{d}^{b} r^{a / d}\right)\right)=\epsilon_{N} \prod_{\substack{a d=N \\ 0 \leq b<d}}\left(f^{(a)}\left(\zeta_{d}^{b} q^{a / d}\right)-f^{(a)}(r)\right) .
$$

2. In [K1, K2], Kozlov studied the relation between complete replicability and modular equations. Cummins and Gannon, using the theory of modular equations, showed that a completely replicable function (satisfying some additional conditions) is a Hauptmodul or a function of the form $q^{-1}+\zeta q$ for $\zeta$ either zero or a twenty-fourth root of 
unity ([CG], Section 8). It would be interesting to study relations between multiplicative symmetries and modular equations. We note that the modular equations for $J(q)$ are characterized by one single multiplicative symmetry ([HKM]).

3. Carnahan ([Ca]) related replicability and complete replicability to Hecke-monicity. It would be also interesting to relate multiplicative symmetries to Hecke-monicity.

\section{Acknowledgement}

The authors would like to thank the referee for carefully reading our manuscript and for giving valuable suggestions.

The first author thanks the Max Planck Institute for Mathematics for support and an invitation in July and August 2017. He also thanks Kyoto Sangyo University and Professor Murase for an invitation in January 2018 to extend our collaboration. He also thanks Prof. Krieg for useful discussions at the Graduate school: Experimental and constructive algebra, at the RWTH Aachen in 2018 and the German University of Technology in Oman for work leave.

The second author is partially supported by Grants-in-Aids from JSPS (17K05186).

\section{References}

[ACMS] D. Alexander, C. Cummins, J. McKay and C. Simons, Completely replicable functions, in Groups, Combinatorics and Geometry, London Math. Soc. Lecture Notes Series 165, 1992, 87-95.

[Bo] R. E. Borcherds, Monstrous moonshine and monstrous Lie superalgebras, Invent. Math. 109 (1992), 405-444.

[Ca] S. Carnahan, Generalized moonshine I: Genus-zero functions, Algebra \& Number Theory 4 (2010), 649-679.

[CN] J. H. Conway and S. P. Norton, Monstrous Moonshine, Bull. London Math. Soc. 11 (1979), 308-339.

[Cu] C. J. Cummins, Some comments on replicable functions, Modern trends in Lie algebra representation theory (Kingston, ON, 1993), 48-55.

[CG] C. J. Cummins and T. Gannon, Modular equations and the genus zero property of moonshine functions, Invent. Math. 129 (1997), no. 3, 413-443. 
[CN] C. J. Cummins and S. P. Norton Rational Hauptmoduls are replicable, Canad. J. Math. 47 (1995), no. 6, 1201-1218.

[Fa] G. Faber, Über Tschebycheffsche Polynome, Crelle 150 (1920) 79-106.

[HKM] B. Heim, C. Kaiser, and A. Murase Modular curves and symmetries of Hecke type, International Journal of Mathematics 29 No. 07 (2018) 1850045.

[K1] D. N. Kozlov, On completely replicable functions and extremal poset theory, M. Sc. thesis, Department of Mathematics, University of Lund, Sweden (1994).

[K2] D. N. Kozlov, On functions satisfying modular equations for infinitely many primes, Cand. J. Math. 51 (1999), 1020-1034.

[Ma] Y. Martin, On modular invariance of completely replicable functions, Contemporary Math. 193 (1996), 263-286.

[N] S. P. Norton, More on Moonshine, Computational group theory (Durham, 1982), 185-193, Academic Press, London, 1984.

[Schi] M. Schiffer, Faber polynomials in the theory of univalent functions, Bulletin of AMS, 54 (1948) 503-517. 